\title{
Optimal and noise-robust extraction of Fracture Mechanics parameters from kinematic measurements
}

\author{
Julien Réthoréa ${ }^{\text {, Stéphane Roux }}{ }^{\mathrm{b}}$, François Hild ${ }^{\mathrm{b}}$ \\ ${ }^{a}$ Laboratoire de Mécanique des Contacts et des Structures (LaMCoS) \\ INSA Lyon / UMR CNRS 5259 \\ 20 Avenue des Sciences, F-69621 Villeurbanne Cedex, France \\ ${ }^{b}$ Laboratoire de Mécanique et Technologie (LMT-Cachan) \\ ENS Cachan / UMR CNRS 8535 / Université Paris 6 / PRES UniverSud Paris \\ 61 Avenue du Président Wilson, F-94235 Cachan Cedex, France
}

\begin{abstract}
The paper is devoted to an optimal (i.e. noise robust) determination of stress intensity factors and crack tip locations based on a displacement field measured over an arbitrarily shaped domain. As the minimization of the noise sensitivity is included within the proposed extraction technique, this is especially dedicated to corrupted displacement fields, e.g. as measured by an optical technique. The main idea is to construct for mode $I$ and $I I$ fields an extracting function so that its $L_{2}$ scalar product with the actual displacement field yields the sought parameter. The extracting function is also constrained to be orthogonal to a set of admissible elastic fields. Two applications are considered to illustrate the technique. The first example deals with a fatigue crack in steel for which small scale yielding occurs. A second example with a
\end{abstract}

Email addresses: julien.rethore@lamcos.insa-lyon.fr (Julien Réthoré), stephane.roux@lmt.ens-cachan.fr (Stéphane Roux), francois.hild@lmt.ens-cachan.fr (François Hild) 
low signal / noise ratio illustrates the capability of the approach to analyze a crack in silicon carbide with sub-pixel openings.

Keywords: Full field measurement, noise sensitivity, stress intensity factors, uncertainty 


\section{Nomenclature}

\begin{tabular}{l|l}
$a_{i}^{n}$ & Amplitude of a displacement field decomposed over Williams' series \\
$\{\mathbf{A}\}$ & Vector representation of $a_{i}^{n}$ \\
$\mathbf{A}^{T}$ & Transpose of matrix or vector A \\
$\{\mathbf{d A}\}$ & Vector representation of perturbation on $a_{i}^{n}$ \\
$\left\{\mathbf{b}^{i}\right\}$ & Vector form of the correlation residual at iteration $i$ \\
{$[\mathbf{C}]$} & Covariance matrix for the displacement uncertainties \\
$D$ & Diameter of the integration domain \\
$E$ & Young's modulus \\
$f$ & Reference image \\
$g$ & Deformed image \\
$G$ & Energy release rate \\
$I^{i n t}$ & Interaction integral \\
$J$ & Rice's $J$-integral \\
$K_{I}^{-1}$ & Dirst mode $I$ super-singular factor \\
$K_{i}$ & Third mode $I$ super-singular factor \\
$K_{I o}$ & Rtress intensity factor in mode $i=I$ or $I I$ \\
$\delta K$ & Peference value for $K_{I}$ \\
\hline $\mathbf{L}]$ & Linking matrix \\
\hline$K_{I}$
\end{tabular}




\begin{tabular}{|c|c|}
\hline $\mathbf{m}$ & Pixel map \\
\hline $\mathbb{M}$ & Set of integer that indexes basis function \\
\hline$[\mathbf{M}]$ & Projection matrix corresponding to $\mathbf{n}_{i}$ functions \\
\hline$[\mathbf{M}]^{-1}$ & Inverse of $[\mathbf{M}]$ \\
\hline$n$ & Integer indexing Williams' fields \\
\hline $\mathbf{n}_{i}$ & Basis functions the experimental displacement field \\
\hline$[\mathbf{N}]$ & Search direction for digital image correlation \\
\hline$p$ & Physical size of a pixel \\
\hline$[\mathbf{P}]$ & Condensed matrix form of the interaction integral \\
\hline q & Virtual crack extension field \\
\hline$r$ & Distance to the crack tip \\
\hline$r_{0}$ & Radius of the masked zone around the crack tip \\
\hline$R$ & Load ratio \\
\hline $\mathcal{R}_{\text {cor }}$ & Correlation residual \\
\hline $\mathcal{R}$ & Extraction residual \\
\hline$\{\mathbf{U}\}$ & Vector representation of the nodal displacement field \\
\hline$\{\mathbf{d U}\}$ & Vector representation of the nodal displacement perturbation \\
\hline $\mathbf{u}$ & Displacement field \\
\hline $\mathbf{u}^{a}$ & Auxiliary displacement field \\
\hline$\left\{\mathbf{U}^{a}\right\}$ & Vector representation of the nodal auxiliary displacement field \\
\hline $\mathbf{u}^{\exp }, u_{i}^{\exp }$ & Experimental displacement field, and $i$-th component \\
\hline$\llbracket u_{1} \rrbracket, \llbracket u_{2} \rrbracket$ & Displacement jumps in the tangential and normal directions, respectively \\
\hline
\end{tabular}




\begin{tabular}{l|l}
{$[\mathbf{V}]$} & Matrix representation of the nodal extractor field \\
$\left\{\mathbf{V}_{1}\right\}$ & Vector representation of the nodal extractor field associated with $a_{1}$ \\
$\left\{\mathbf{v}^{i n t}\right\}$ & Extractor vector field associated with $I^{\text {int }}$ \\
$\left\{\mathbf{V}^{i n t}\right\}$ & Vector representation of the nodal extractor field associated with $I^{\text {int }}$ \\
$\mathbf{x}_{i}$ & Local coordinate system at the crack tip $(i=1,2)$ \\
$x_{I}$ & Shift of the tip from its actual position along $\mathbf{x}_{1}$ \\
$\mathbf{z}$ & Position of a point in the complex plane \\
$\Gamma$ & Crack path \\
$\boldsymbol{\delta}$ & Kronecker symbol \\
$\{\boldsymbol{\Delta}\}$ & Vector representation of $\delta_{i 1}$ \\
$\kappa$ & Kolossov's constant \\
$\{\boldsymbol{\Lambda}\}$ & Vector representation of Lagrange multipliers \\
$\mu$ & Lamé's coefficient \\
$\nu$ & Poisson's ratio \\
$\sigma_{c}$ & Standard deviation of image noise \\
$\boldsymbol{\sigma}$ & Standard deviation of sensor noise \\
$\sigma_{y}$ & Auxiliary stress tensor associated with $\mathbf{u}^{a}$ \\
\hline
\end{tabular}




\begin{tabular}{l|l}
$\boldsymbol{\phi}_{I}^{n}$ & Mode $I$ fields in Williams' series \\
$\boldsymbol{\phi}_{I I}^{n}$ & Mode $I I$ fields in Williams' series \\
$\varphi_{i k}^{j}$ & $\boldsymbol{\phi}_{i}^{j}$ functions projected on the interpolation basis $\mathbf{n}_{k}$ \\
{$[\boldsymbol{\Phi}]$} & $\begin{array}{l}\text { matrix that collects in its rows the components of all } \boldsymbol{\phi}_{i}^{j} \text { functions } \\
\text { projected on the interpolation basis } \mathbf{n}_{k}\end{array}$ \\
$\boldsymbol{\psi}_{i}$ & Extracting functions \\
$\left\{\boldsymbol{\Psi}_{i}\right\}$ & Vector representation of the nodal extracting function \\
$\theta$ & Polar angle of the current point with respect to the crack tip axis \\
$\tau_{K}$ & Noise sensitivity of mode $I$ stress intensity factor \\
$\tau_{K}-1$ & Noise sensitivity of mode $I$ first super singular factor \\
$\Omega$ & Integration domain \\
$\partial \Omega$ & Boundary of the integration domain \\
$\langle\ldots\rangle$ & Average value \\
$\nabla$ & Gradient operator
\end{tabular}




\section{Introduction}

One of the central issues of Fracture Mechanics is to evaluate global parameters (e.g. stress intensity factors) from a given displacement field. This is an important question for numerical modeling $[18,1,7,19,16]$ and also an essential problem for experimental characterization [14, 12, 6, 21, 28, 32] of cracks. One of the main difficulties of this problem is the issue of scale, namely, not too large (to avoid structural effects) but not too small (to keep clear from the inelastic behavior, or numerical discretization biases). An additional difficulty arises when the displacement field is extracted from experimental data that are corrupted by measurement uncertainties. Minimizing the effect of this noise on the determination of Fracture Mechanics parameters is a key property, which is addressed in the present study.

Two approaches of Linear Elastic Fracture Mechanics are usually distinguished, namely, the energy and the kinematic (or static) approaches. The energy release rate $G[8]$ and $J$-integral [27] and are two complementary methods that evaluate the amount of energy released in a virtual extension of the crack. The kinematic approach defines the stress intensity factors [13] for a 2D solid as a limit

$$
K_{I} \propto \lim _{r \rightarrow 0} \mu \sqrt{\frac{2 \pi}{r}} \llbracket u_{2} \rrbracket \quad K_{I I} \propto \lim _{r \rightarrow 0} \mu \sqrt{\frac{2 \pi}{r}} \llbracket u_{1} \rrbracket
$$

In 2D linear elasticity, the following result

$$
J=G \propto \frac{K_{I}^{2}+K_{I I}^{2}}{\mu}
$$


unifies the two approaches. One of the properties of these quantities is their quadratic dependence in the displacement field and the stress intensity factors respectively. From this observation, a class of methods devoted to mixed mode separation has been developed (see, e.g. Refs. [17, 5, 4]). Among those, the interaction integral reads as a trilinear form of the actual displacement field $\mathbf{u}$, an auxiliary field $\mathbf{u}^{a}$ and a virtual crack extension (VCE) field $\mathbf{q}$

$$
I^{i n t}=-\int_{\Omega}\left[\sigma_{m l}^{a} u_{m, l} \delta_{k j}-\left(\sigma_{i j}^{a} u_{i, k}+\sigma_{i j} u_{i, k}^{a}\right)\right] q_{k, j} \mathrm{~d} \Omega
$$

where Einstein's convention is used, The auxiliary field is chosen to extract separately mode $I$ and $I I$ stress intensity factors. Usually, the asymptotic crack tip fields in modes $I$ and $I I$ are used to obtain $K_{I}$ and $K_{I I}$, respectively. The VCE field is constrained by a few assumptions. First, it must vanish outside $\Omega$, be equal to $x_{1}$ at the crack tip and be parallel to $x_{1}$ along the crack faces. For a given choice of $\mathbf{q}, I^{\text {int }}$ is a bilinear symmetric positive definite form. Thus the interaction integral may be viewed as a scalar product related to the energy norm defined by $J$ or $G$. The stress intensity factors are then estimated from this energy-based scalar product between the actual and auxiliary displacement fields.

The most popular stress intensity factor extracting methods dedicated to experimental applications is based on least squares minimization of the gap between the asymptotic crack tip field and the actual displacement field. One focuses on the crack opening displacement only [6] or on full fields [14, 28, 32]. The interaction integral has recently been used to measure stress intensity 
factors [21] and optimized [24] to reduce the influence of measurement uncertainties on the determination of the sought parameter. The reduction of the noise sensitivity is also one of the aims of the approach proposed herein.

In the present paper, it is shown that all these techniques, interaction integrals as well as least squares extraction, can be recast within the framework detailed in Section 2. It will be shown that the optimized version of the interaction integral [24] and least squares extraction are local optima with respect to noise sensitivity. The technique proposed herein allows for the numerical determination of an optimal extracting function. Theoretical crack displacement fields and corresponding Fracture Mechanics parameters are detailed in Section 2 as well as the formulation of an optimal noise-robust extractor. A numerical resolution is also proposed in this section. Then the Digital Image Correlation (DIC) algorithm used herein to measure displacement fields is briefly presented to derive its noise sensitivity in Section 3. Last, in Section 4, two examples are discussed. The first one is dedicated to a fatigue crack in a steel sample where a confined plastic zone exists at the crack tip. Stress intensity factors and crack tip position are estimated using the optimal extraction of appropriate crack displacement field parameters. Second, the extraction of stress intensity factors is examined from a low signal-to-noise ratio displacement field of a brittle material. 


\section{Problem formulation}

\subsection{Crack displacement fields}

Let us consider a semi-infinite planar crack whose normal is aligned along the $\mathbf{x}_{2}$ axis, and crack front parallel to the $\mathbf{x}_{3}$ axis. The crack corresponds to the domain $x_{2}=0$ and $x_{1}<0$. Crack displacement fields $\mathbf{u}$ in the linear elastic regime are computed as a double infinite series of KolossovMuskhelishvili (K \& M) functions [31] $\phi_{I}^{n}$ and $\phi_{I I}^{n}$ for modes I and II. The complex plane formulation reveals convenient in the present case. A point $\mathbf{z}$ in the complex plane $x_{1}+i x_{2}$ is represented in polar coordinates $(r, \theta)$ such that $\mathbf{z}=r e^{i \theta}$. The crack tip is located at the origin, $\mathbf{z}=\mathbf{0}$, and the semi-infinite crack extends in the direction $\theta= \pm \pi$.

The displacement field $\mathbf{u}$ is conventionally represented by its complex writing, $\mathbf{u}=u_{1}+i u_{2}$. It is expanded as a double series

$$
\mathbf{u}(r, \theta)=\sum_{i=I, I I} \sum_{n} a_{i}^{n} \boldsymbol{\phi}_{i}^{n}(r, \theta)
$$

where $a_{i}^{n}$ are the sought parameters, and $\phi_{i}^{n}$ reference fields that form the complete set of linear elastic fields satisfying a condition of zero traction along the crack path:

- even $n$

$$
\begin{aligned}
\phi_{I}^{n}(r, \theta) & =r^{n / 2}\left(\kappa e^{i n \theta / 2}-\frac{n}{2} e^{i(4-n) \theta / 2}+\left(\frac{n}{2}+1\right) e^{-i n \theta / 2}\right) \\
\phi_{I I}^{n}(r, \theta) & =i r^{n / 2}\left(\kappa e^{i n \theta / 2}+\frac{n}{2} e^{i(4-n) \theta / 2}-\left(\frac{n}{2}-1\right) e^{-i n \theta / 2}\right)
\end{aligned}
$$


- odd $n$

$$
\begin{gathered}
\phi_{I}^{n}(r, \theta)=(-1)^{(n+1) / 2} r^{n / 2}\left(\kappa e^{i n \theta / 2}-\frac{n}{2} e^{i(4-n) \theta / 2}+\left(\frac{n}{2}-1\right) e^{-i n \theta / 2}\right) \\
\phi_{I I}^{n}(r, \theta)=i(-1)^{(n-1) / 2} r^{n / 2}\left(\kappa e^{i n \theta / 2}+\frac{n}{2} e^{i(4-n) \theta / 2}-\left(\frac{n}{2}+1\right) e^{-i n \theta / 2}\right)
\end{gathered}
$$

where $\kappa$ is Kolossov's constant, $\kappa=(3-\nu) /(1+\nu)$ for plane stress or $\kappa=(3-4 \nu)$ for plane strain conditions.

The partition between mode I and II is performed on the basis of the following symmetries

$$
\begin{aligned}
& \phi_{I}^{n}(\overline{\mathbf{z}})=\overline{\phi_{I}^{n}(\mathbf{z})} \\
& \phi_{I I}^{n}(\overline{\mathbf{z}})=-\overline{\phi_{I I}^{n}(\mathbf{z})}
\end{aligned}
$$

All these fields are self-similar, and thus are homogeneous functions of the distance to the crack tip $r$. It is convenient to label them with the integer index $n$ that indicates the exponent of the power-law dependence of the displacement with $r$, that is $n / 2$. With this convention, all elastic displacement fields are generated with $-\infty<n<\infty$. All odd indices label fields with a discontinuity across the crack mouth, whereas even indices correspond to continuous fields. To mention just a few examples, $n=0$ corresponds to translations either parallel (hence termed I) or perpendicular (labeled II) to the crack path, $n=1$ are the classical mode I and mode II displacement fields whose amplitudes are proportional to the corresponding stress intensity factors. The case $n=2$ gives access to either the T-stress component for mode I, or rigid body rotation about the crack tip for mode II 
(no discontinuity is involved here since 2 is even). Larger indices correspond to "subsingular" fields that may capture the remote heterogeneity of the loading, but do not affect the fracture behavior at the crack tip.

Classically, i.e. within the framework of Linear Elastic Fracture Mechanics, only $n \geq 0$ fields are considered, since negative indices would correspond to physically unrealistic displacement fields with a diverging elastic energy density at the crack tip. However, inelastic behaviors are typically activated in the vicinity of the crack tip, in the so-called process zone, so that the above linear elastic decomposition does not hold. If a small domain close to the crack tip that contains all non-linearities is omitted, the displacement field in the complementary domain will naturally contain contributions from such $n<0$ fields $[9,25,10]$ (referred to as "supersingular" in the sequel). They are necessary to account for the most general problem where both the small and large $r$ regions are omitted from the elastic description.

\subsection{Fracture Mechanics parameters}

Considering the expression of the general crack tip field in Equations (5) to (8), the following recurrence formulas are obtained

$$
\frac{\partial \phi_{j}^{n}}{\partial x_{1}}=-\frac{n}{2} \boldsymbol{\phi}_{j}^{n-2}
$$

The derivative with respect to the crack tip position $x_{c}$ being the opposite of the above equation, it shows that a mis-positioning of the crack tip along its axis will give rise to a supersingular contribution $\phi_{j}^{-1}$, away from the immediate vicinity of the crack tip. Therefore, in order to estimate the crack 
tip position, the displacement field is decomposed by using $n=1$ and $n=-1$ order functions

$$
a_{j}^{1} \phi_{j}^{1}+a_{j}^{-1} \phi_{j}^{-1}=a_{j}^{1}\left(\phi_{j}^{1}+2 \frac{a_{j}^{-1}}{a_{j}^{1}} \frac{\partial \phi_{j}^{1}}{\partial x_{c}}\right)
$$

For the symmetric part of $\mathbf{u}$, i.e. for mode $I$, this result is interpreted as a Taylor expansion of the usual crack tip field whose tip would be shifted from the actual one by an offset distance $x_{I}$ such that

$$
x_{I}=2 \frac{a_{I}^{-1}}{a_{I}^{1}}
$$

Calculating $x_{I}$ from $a_{I}^{-1}$ (i.e. $\left.\propto K_{I}^{-1}\right)$ and $a_{I}^{1}$ (i.e. $\propto K_{I}$ ), an estimate of the offset between the assumed crack tip position and the actual one is obtained.

More generally, if confined plasticity develops at the crack tip, the above decomposition does not hold down to the crack tip. However, excluding the plastic region (or a larger one) around the crack tip allows one to resort to the above decomposition for the elastic displacement. Hence, the above described procedure leads to a unique and consistent definition of the "equivalent elastic crack tip," i.e. the one that would provide the most accurate correspondence between the displacement field of the medium under study and that of a cracked homogeneous elastic medium with the same stress intensity factor. This strategy straightforwardly applies to any order function. The proper interpretation of this quantity is based on the analysis of a domain where all crack tip non-linearities have been cut out. The elastic description, which contains any negative order (supersingular) field contribution, can thus be 
extrapolated down to the (elastic equivalent) crack tip. By construction, or definition of the crack tip, the $n=-1$ or dipolar crack amplitude vanishes. The quadrupolar term $(n=-3)$ is the first non vanishing intrinsic characteristic of the crack strain field. For all types of non-linearity (e.g. plasticity, damage, cohesive crack tip) the amplitude $a^{-3}$ exists, and provides a unique and well defined scalar signature of the non-linearities. Higher order terms are associated with fields that vanish more quickly away from the crack tip.

To summarize, for a complete identification of the mechanical state of a material around the crack tip, first a correct position is searched for. Second, stress intensity factors are estimated using the estimated crack tip position. This procedure may be performed iteratively.

\subsection{Extraction problem}

Let us consider that the displacement field, $\mathbf{u}$, is measured on the surface of a cracked sample. This experimentally measured displacement field is decomposed onto an interpolation basis

$$
\mathbf{u}^{\exp }\left(x_{1}, x_{2}\right)=\sum_{i \in \mathbb{M}} u_{i}^{\exp } \mathbf{n}_{i}\left(x_{1}, x_{2}\right)
$$

In the domain where non-linearities can be neglected, the actual displacement field, $\mathbf{u}$, theoretically belongs to the space generated by the basis functions $\{\boldsymbol{\phi}\}$ as a solution to a Linear Elastic Fracture Mechanics problem. Hence, amplitudes $a_{i}^{j}$ are introduced such that [see Equation (4)]

$$
\mathbf{u}\left(x_{1}, x_{2}\right)=\sum_{j}\left[a_{I}^{j} \phi_{I}^{j}\left(x_{1}, x_{2}\right)+a_{I I}^{j} \boldsymbol{\phi}_{I I}^{j}\left(x_{1}, x_{2}\right)\right]
$$


The (best) determination of amplitudes $a_{i}^{j}$ - collectively designated by the vector form $\{\mathbf{A}\}$ - is the objective of the present paper.

The measured displacement field is restricted to the interpolation space generated by the function basis $\mathbf{n}_{i}$, and, in the case of digital image correlation, it is itself obtained by a least squares minimization of an $L_{2}$ norm as recalled in the next Section. Introducing the "mass matrix"

$$
M_{i j}=\int_{\Omega} \mathbf{n}_{i}\left(x_{1}, x_{2}\right) \cdot \mathbf{n}_{j}\left(x_{1}, x_{2}\right) \mathrm{d} \Omega
$$

the measured displacement field reads

$$
\mathbf{u}^{\exp }\left(x_{1}, x_{2}\right)=M_{i j}^{-1}\left(\int_{\Omega} \mathbf{n}_{j}\left(x_{1}, x_{2}\right) \cdot \mathbf{u}\left(x_{1}, x_{2}\right) \mathrm{d} \Omega\right) \mathbf{n}_{i}\left(x_{1}, x_{2}\right)
$$

where Einstein's convention is used. $\mathbf{u}^{\exp }$ is thus expressed as

$$
\mathbf{u}^{\exp }\left(x_{1}, x_{2}\right)=\left(a_{I}^{j} \varphi_{I i}^{j}+a_{I I}^{j} \varphi_{I I i}^{j}\right) \mathbf{n}_{i}\left(x_{1}, x_{2}\right)
$$

where $\varphi_{i k}^{j}$ can be rewritten in matrix form $[\mathbf{\Phi}]$ that collects in its rows the components of all $\phi_{i}^{j}$ functions projected on the interpolation basis $\mathbf{n}_{k}$.

To estimate amplitudes $\{\mathbf{A}\}$, it is proposed to introduce a set of extracting functions $\{\boldsymbol{\psi}\}$ in the interpolation space so that

$$
\int_{\Omega} \boldsymbol{\psi}_{i}\left(x_{1}, x_{2}\right) \cdot \Phi_{j k} \mathbf{n}_{k}\left(x_{1}, x_{2}\right) \mathrm{d} \Omega=\delta_{i j}
$$

Numerically, it is more convenient to work with amplitudes $\Psi_{i q}$ of $\boldsymbol{\psi}_{i}$ functions on $\mathbf{n}_{q}$ interpolation functions, or $\boldsymbol{\psi}_{i}\left(x_{1}, x_{2}\right)=\Psi_{i q} \mathbf{n}_{q}\left(x_{1}, x_{2}\right)$. The above conjugation property is rephrased as

$$
\Psi_{i q}\left(\int_{\Omega} \mathbf{n}_{q}\left(x_{1}, x_{2}\right) \cdot \mathbf{n}_{k}\left(x_{1}, x_{2}\right) \mathrm{d} \Omega\right) \Phi_{j k}=\delta_{i j}
$$


For the set of functions $\{\boldsymbol{\psi}\}$, their scalar product with the measured displacement field yields the following identity

$$
\int \boldsymbol{\psi}_{i}\left(x_{1}, x_{2}\right) \cdot \mathbf{u}^{\exp }\left(x_{1}, x_{2}\right) \mathrm{d} \Omega=a_{i}
$$

or

$$
\{\mathbf{A}\}=[\mathbf{\Psi}][\mathbf{M}]\{\mathbf{U}\}
$$

where $\{\mathbf{U}\}$ collects all $u_{k}^{\exp }$. For numerical implementation, it is convenient to compute matrix $[\mathbf{V}]=[\mathbf{M}][\mathbf{\Psi}]^{T}$, so that the extraction of $\{\mathbf{A}\}$ is given by the following product

$$
\{\mathbf{A}\}=[\mathbf{V}]^{T}\{\mathbf{U}\}
$$

For this reason, $[\mathbf{V}]$ are referred to as "extractors" or extracting functions. The present procedure is general and may be tailored to measure as many amplitudes $a_{i}$ as there are degrees of freedom in the measured displacement field. However, in practice, a lack of accuracy will hinder the performance of the extraction. Thus only a limited set of functions $\phi$ and $\boldsymbol{\psi}$ will be considered. As a consequence, the conjugation property (19) is not sufficient to compute the extractors. It is proposed to supplement these conditions with another type of constraint, i.e. noise-robustness, as detailed in the following section. A similar approach was also proposed in the framework of the virtual fields method [2].

\subsection{Noise robustness}

Being obtained from experimental data (e.g. images), $\mathbf{u}^{\exp }$ is inevitably corrupted by measurement uncertainties, whose influence on the extracted 
coefficients is to be reduced as much as possible. A perturbation vector of the component of $\mathbf{u}^{\exp }$ in the interpolation basis is denoted by $\{\mathbf{d} \mathbf{U}\}$. This perturbation is defined as a zero average noise

$$
\langle\{\mathbf{d U}\}\rangle=\{\mathbf{0}\}
$$

with the following correlation kernel

$$
\left\langle\{\mathbf{d U}\}\{\mathbf{d U U}\}^{T}\right\rangle=[\mathbf{C}] \sigma^{2}
$$

when the inputs (i.e. the images) of the correlation algorithm are affected by a spatially uncorrelated noise with a standard deviation $\sigma$. Section 3 is devoted to the analysis of correlation kernel $[\mathbf{C}]$.

The displacement uncertainty subsequently affects the extracted coefficients $a_{i}$, and by using Equation (22), the corresponding perturbation reads

$$
\{\mathbf{d A}\}=[\mathbf{V}]^{T}\{\mathbf{d} \mathbf{U}\}
$$

Consequently, $\{\mathbf{d} \mathbf{A}\}$ is also of zero mean and its variance becomes

$$
\left\langle\{\mathbf{d A}\}\{\mathbf{d A}\}^{T}\right\rangle=[\mathbf{V}]^{T}[\mathbf{C}][\mathbf{V}] \sigma^{2}
$$

This result quantifies the effect of measurement noise on the extracted coefficients. Noise robustness is achieved through the minimization of the above quantity. For instance, one single amplitude (e.g. stress intensity factor $\left.K_{I} \propto a_{I}^{1}\right)$ may be searched for. In that case, only $\left\langle d K_{I}\right\rangle$ is to be minimized. If several quantities are to be estimated, the minimization of each variance leads to the determination of the corresponding extractor. 


\subsection{Optimal extraction}

The procedure of optimal extraction is based on the joint use of the conjugation property (19) and the noise robustness condition (26). Let us consider that $a_{1}$ is searched for. Only a reduced set of meaningful elastic fields will be considered, namely $i \in\left[n_{0} ; n_{1}\right]$, so that the conjugation conditions are written as

$$
\Psi_{1 k} M_{k j} \Phi_{i j}=\delta_{1 i}
$$

Reverting to $\left\{\mathbf{V}_{1}\right\}$ vector, the above equations are recast as

$$
V_{1 j} \Phi_{i j}=\delta_{1 i}
$$

Under these constraints, the minimum of

$$
\mathcal{R}=\frac{1}{2} V_{1 i} C_{i j} V_{j 1}
$$

is sought. This constrained minimization problem is solved by using Lagrange multipliers denoted by $\{\boldsymbol{\Lambda}\}$

$$
\left[\begin{array}{cc}
{[\mathbf{C}]} & {[\boldsymbol{\Phi}]^{T}} \\
{[\mathbf{\Phi}]} & {[\mathbf{0}]}
\end{array}\right]\left\{\begin{array}{c}
\left\{\mathbf{V}_{\mathbf{1}}\right\} \\
\{\boldsymbol{\Lambda}\}
\end{array}\right\}=\left\{\begin{array}{c}
\{\mathbf{0}\} \\
\{\boldsymbol{\Delta}\}
\end{array}\right\}
$$

It is worth noting that if $[\mathbf{C}]$ is proportional to the identity matrix, i.e. if the noise is considered as uncorrelated and uniform (white noise), then the optimal extractor is exactly the least squares extractor. Thus least squares extraction is the best extractor for a white noise.

As mentioned in the introduction, the interaction integral [21, 24] can be read as a scalar product between the actual displacement and an auxiliary 
displacement with respect to a given norm involving an interaction Eshelby tensor integrated over the domain affected by a virtual extension of the crack. In the displacement functional basis, the interaction integral is rewritten in a matrix-vector format as

$$
I^{\text {int }}=\left\{\mathbf{U}^{a}\right\}^{T}[\mathbf{P}]\{\mathbf{U}\}
$$

Thus, if $\left\{\mathbf{V}^{\text {int }}\right\}=[\mathbf{P}]\left\{\mathbf{U}^{a}\right\}, I^{\text {int }}$ is computed in a similar formalism as $\{\mathbf{A}\}$ and interaction integrals can be recast in the framework proposed herein. They are associated with a given extracting field $\mathbf{v}^{\text {int }}$. Due to the Eshelbian norm in the interaction integral, its associated extracting field is in the subspace of fields that satisfy the extraction conditions. The specific form of the weight function introduces restrictions on the associated extractor field, so that the sub-space to which it belongs is much smaller than the general case considered herein. The optimal version proposed in Ref. [24] is thus a local minimizer of the noise sensitivity. This property will be illustrated in the example section.

Up to now, the nature of domain $\Omega$ was not specified precisely. The only (implicit) property is that it should only contain a region of space where linear elasticity holds. However, it may contain holes, it may not include the crack mouth, it may have a complicated boundary, and the above formulation will still hold. Such a tolerant formulation is suited to the exploitation of image correlation data where information may be lacking in some regions. This is to be contrasted with the interaction integral formalism that requires 
at least a good capturing of the displacements along the crack faces.

The present framework will be applied to two examples dealing with displacement fields measured by DIC, which is briefly summarized in the next section.

\section{Digital image correlation}

In the following, 2D displacement measurements are considered by using pictures $f$ and $g$ of a sample surface. The passive advection of the texture between the reference $f$ and deformed $g$ images reads

$$
f(\mathbf{m})=g\left(\mathbf{m}+\mathbf{u}^{\exp }(\mathbf{m})\right)
$$

over the entire pixel map $\mathbf{m}$. From the knowledge of $f$ and $g$, the measurement problem consists in identifying $\mathbf{u}^{\exp }$ as accurately as possible. As such, the problem is ill-posed, unless additional assumptions are made on the displacement field so that the information is sufficient to determine $\mathbf{u}^{\text {exp }}$ with a reasonable accuracy. The quadratic difference is integrated with respect to m over a domain $\Omega$ (i.e. a Galerkin approach to the measurement problem)

$$
\mathcal{R}_{\text {cor }}=\int_{\Omega}\left[f(\mathbf{m})-g\left(\mathbf{m}+\mathbf{u}^{\exp }(\mathbf{m})\right)\right]^{2} \mathrm{~d} \Omega
$$

and considered as a residual $\mathcal{R}_{\text {cor }}$ to be minimized with respect to the set of kinematic unknowns. Adopting a general discretization scheme of the displacement field (see Equation (13)) allows for the measurement of the degrees of freedom $u_{i}^{\text {exp }}$. 
At this level of generality, many choices can be made to measure 2D displacement fields. When 2D images are considered, 4-noded elements defined on a square grid (i.e. bi-linear functions of $x_{1}$ and $x_{2}$ [33]) may be chosen (it is referred to as Q4-DIC [3]). Enriched kinematics (à la X-FEM [15]) may also be considered to describe weak or strong discontinuities associated with Q4 elements (i.e. XQ4-DIC $[22,20]$ ). In all the previous cases, no hypotheses are made concerning the underlying material behavior. When the latter is known (e.g. linear and isotropic elasticity), integrated approaches can be developed to directly evaluate mechanically significant quantities (e.g. elastic properties [11] or even stress intensity factors $[28,9,25])$. This last route is not followed hereafter, rather so-called Q4 and XQ4 kinematics are measured and subsequently post-processed.

The interpolation functions being chosen, the minimization of $\mathcal{R}_{\text {cor }}$ is carried out using a modified Newton procedure

$$
[\mathbf{N}]\left\{\mathbf{U}^{i}\right\}=\left\{\mathbf{b}^{i}\right\}
$$

that is iteratively solved until convergence is reached. The following definition holds for the search direction $[\mathbf{N}]$ of the modified Newton procedure and the residual $\left\{\mathbf{b}^{i}\right\}$ at iteration $i$

$$
N_{k l}=\int_{\Omega}\left(\mathbf{n}_{k}(\mathbf{m}) \cdot \nabla f(\mathbf{m})\right)\left(\mathbf{n}_{l}(\mathbf{m}) \cdot \nabla f(\mathbf{m})\right) \mathrm{d} \Omega
$$

and

$$
b_{k}^{i}=\int_{\Omega}\left(\mathbf{n}_{k}(\mathbf{m}) \cdot \nabla f(\mathbf{m})\right)\left(f(\mathbf{m})-g\left(\mathbf{m}+\left(\mathbf{u}^{\exp }(\mathbf{m})\right)^{i}\right)\right) \mathrm{d} \Omega
$$


The gradient of the image as well as sub-pixel interpolations have been the subject of publications in the literature [30, 29]. A standard choice is a finite difference gradient and cubic spline interpolation.

Let us now consider that the images are affected by noise. It is assumed that $f$ is a pure (noise free) reference image and noise is reported on $f-$ g. This noise is considered as spatially uncorrelated, of zero mean and its variance is equal to twice the variance of the sensor noise $\sigma_{c}^{2}$. Typical values for $\sigma_{c}$ are 1 or 2 gray levels for 8-bit pictures. As a consequence, the image noise is reported on the LHS $\left\{\mathbf{b}^{i}\right\}$ of Equation (34) for which the variance reads

$$
\left\langle b_{k}^{i} b_{l}^{i}\right\rangle=2 \sigma_{c}^{2} \int_{\Omega}\left(\mathbf{n}_{k}(\mathbf{m}) \cdot \nabla f(\mathbf{m})\right)\left(\mathbf{n}_{l}(\mathbf{m}) \cdot \nabla f(\mathbf{m})\right) \mathrm{d} \Omega
$$

The search direction $[\mathbf{N}]$ then leads to the displacement perturbation

$$
\left\langle\{\mathbf{d U}\}\{\mathbf{d} \mathbf{U}\}^{T}\right\rangle=2 \sigma_{c}^{2}[\mathbf{N}]^{-1}[\mathbf{N}][\mathbf{N}]^{-1}
$$

The correlation kernel of the displacement uncertainty is identified as

$$
[\mathbf{C}] \sigma^{2}=2 \sigma_{c}^{2}[\mathbf{N}]^{-1}
$$

Covariance matrix $[\mathbf{C}]$ is directly related to the image texture (i.e. its gradient) and the chosen measurement basis $\mathbf{n}_{k}$. The joint use of the last results (Equations (39) and (30)) allows for the computation of the optimal extractor precisely suited to a specific image, and a chosen kinematic basis. 


\section{Examples}

The present section shows the application of the above formalism to two examples. In the first one - a fatigue test on a steel specimen - the above described procedure is used to identify the crack tip position and stress intensity factors. The second one deals with a brittle ceramic material for which a very low signal-to-noise ratio is observed. In that case, the extraction of the mode $I$ stress intensity factor is addressed, and a comparison is performed with alternative techniques.

\subsection{Fatigue test on a steel CCT specimen}

This first example is devoted to the analysis of a CCT specimen made of XC48 (or C45) steel with Young's modulus of $190 \mathrm{GPa}$ and Poisson's ratio of 0.3. The cyclic yield strength $\sigma_{y}$ (offset: $0.2 \%$ ) is equal to $210 \mathrm{MPa}$. The specimen is subjected to cyclic tension with a load ratio $R=0.4$. In the present analysis, only the stage corresponding to the maximum load level is considered after about 300,000 cycles for which the crack size $2 a=$ $14.5 \mathrm{~mm}$. The sample thickness is equal to $5 \mathrm{~mm}$. The vertical and horizontal components of the displacement field obtained by using $12 \times 12$-pixel Q4 elements are shown in Figure 1. The conversion factor $p$ is $2.08 \mu \mathrm{m} /$ pixel.

In this example, a process zone is expected. The exact position of the crack tip along the crack mouth is searched for. This location is critical if a stress intensity factor is to be evaluated [28]. Thus the supersingular component $K_{I}^{-1}$ has to be extracted. It allows for an estimate of the crack 
tip location $[9,25,10]$. Further, a mask is adopted to account for linear elastic displacement fields, i.e. a 20-pixel wide band and a core 20 pixels in radius are omitted for the extraction. In the sequel, the domain size is the diameter of the disk that defines the outer boundary of the mask.

\subsubsection{Crack tip location}

In Figure 2(a), the estimation of the shift $x_{I}$ with respect to the prescribed position of the crack tip along the crack faces is plotted. A linear dependence is obtained with a -1 slope. From Equation (12), the ratio between the shift of the crack tip $x_{I}$ and the crack tip position used to compute $\mathrm{K} \&$ M potentials is theoretically equal to -1 . This is confirmed by Figure 2(a) for both optimal and least squares extraction techniques, the latter being 1 pixel or about $2 \mu \mathrm{m}$ higher. The variation of the crack tip shift (and thus of $K_{I}^{-1}$ ) is evaluated by using the results of Figure 2(b). A typical variation of $5 \mu \mathrm{m}$ is

obtained for both extraction techniques. The range within which $K_{I}^{-1}$ is less dependent on the domain size appears lower than the independence range of the stress intensity factors (see Figure 3(a)), which could have been expected considering the higher variation of the first supersingular field compared with the usual $\sqrt{r}$-dependence of the near crack tip field.

Figure 2(c) shows the noise sensitivity of the extraction of $K_{I}^{-1}$. As observed for the stress intensity factor, the optimal extraction allows for a reduction of this noise sensitivity compared with the results obtained with the least squares approach. Considering that the noise sensitivity of $K_{I}$ 
has only a small contribution to the noise sensitivity of the identification of the crack tip position, the uncertainty level of the shift estimation is about $0.03 \mu \mathrm{m} /$ gray level for the optimal extraction.

\subsubsection{SIF extraction}

The results on the stress intensity factor extraction and their noise sensitivity are presented in Figure 3. In the present case, the signal-to-noise ratio is high. Consequently, least squares and optimal extractions give very similar results for $K_{I}$ and $K_{I I}$, namely, a value of $20.7 \mathrm{MPa} \sqrt{\mathrm{m}}$ is obtained for $K_{I}$, and $0.05 \mathrm{MPa} \sqrt{\mathrm{m}}$ for $K_{I I}$. Because of the high signal-to-noise ratio, a very low dependence to the size of the integration domain is observed as well as very low noise sensitivity. A $0.0011 \mathrm{MPa} \sqrt{\mathrm{m}} /$ gray level standard uncertainty is obtained for the least squares extraction, and 0.0009 $\mathrm{MPa} \sqrt{\mathrm{m}} /$ gray level for the optimal approach.

\subsubsection{Extracting fields}

Figures 4-5 show the components of the extracting fields for $K_{I}^{1}$ and $K_{I}^{-1}$ using least squares and optimal extractions. As for $K_{I}$, the optimal fields spread the weight allowed to each displacement degree of freedom depending on its associated level of confidence. First, due to the divergence of supersingular fields, $n=-1$, in the vicinity of the crack tip, their associated extracting fields weight more intensively the displacement data around the core of the mask. Second, the angular dependence, bipolar for $n=-1$, could have been derived from the expression of the supersingular fields. Compared 
with extracting fields of stress intensity factors, the latter ones are more concentrated in the vicinity of the crack leading to an expected and observed higher sensitivity to noise.

\subsection{Sandwiched beam experiment on a SiC specimen}

This second example is dedicated to the extraction of mode $I$ stress intensity factors in a sandwiched beam experiment [6] on a silicon carbide sample. The linear elastic behavior of $\mathrm{SiC}$ is characterized by Young's modulus $E=410 \mathrm{GPa}$ and Poisson's ratio $\nu=0.15$. The crack tip positioning is crucial since the values of stress intensity factors are significantly dependent on the former [28]. First, the analysis is carried out in order to extract the supersingular contribution in the displacement field. Then, we focus on the extraction of $K_{I}$ and we compare different techniques including the interaction integral method.

The region of interest covers an area of $706 \times 646$ pixels approximately centered about the crack tip. The displacement field is measured by using $16 \times 16$-pixel Q4 elements with a discontinuous enrichment of the kinematic basis [23]. Figure 6 shows the two components of the measured displacement field. Due to the poor quality of the image (the rough surface of the material is used as a random texture for image correlation), and also the small magnitude of the displacement fluctuations, a low signal-to-noise ratio is ob-

served. A normal displacement discontinuity of about 0.5 pixel (at most) is captured at the bottom of the region of interest (i.e. the crack is invisible). 
The conversion factor $p$ is $1.85 \mu \mathrm{m} /$ pixel.

\subsubsection{Extraction analysis and $3 D$ effects}

In this study, only the optimal extractor with an $L_{2}$ projection is used. A mask with a disk of radius $r_{0}=20$ pixels, in addition to a 20 pixel wide masked zone around the crack surface is adopted. Crack tip shift and process zone width are shown in Figure 7. Compared to the results of the previous section, the extracted quantities suffer from fluctuations of higher magnitude when the domain size is increasing. A value of the crack tip shift with respect to its initial position of about $-67 \mu \mathrm{m}$ is obtained. When the change of the crack tip shift with respect to the assumed crack tip position is observed, the expected linear dependence is rather well retrieved.

Different arguments can be invoked in order to explain these unexpected results. The first point is that the displacement field is measured on a free surface, and hence the analytic 2D plane stress displacement field that is used is not the actual one. A second point is that a post-mortem observation of the specimen and the crack surface (Figure 8) shows that the crack front is not perfectly orthogonal to the free surfaces on which the displacement was measured in the correlation analysis. Thus, an additional $3 \mathrm{D}$ effect is expected. To evaluate its influence, a 3D elastic analysis is carried out on the entire specimen with idealized crack geometry and boundary conditions. A planar crack is considered and the front is modeled as a parabola such that the crack length on the free surface is half of the specimen width and $180 \mu \mathrm{m}$ 
longer in the middle section, consistent with post-mortem observations (Figure 8). A straight front (normal to the observation face) is also considered as a reference case.

The results are shown in Figures 9 and 10. In Figure 9, the displacement field on the free surface for a straight crack front is depicted. For this analysis, the element size in the region of interest is equal to $16 \times 16$ pixels (for a pixel size of $2.11 \mu \mathrm{m})$. In Figure 10(b), the same quantities as for Figure 7(b) are given as functions of the domain size. Three cases are considered. First, a straight crack front is used and the extraction analysis is carried out on the free surface and also on the mid-plane of the specimen (under plane strain conditions). Second, the displacement field on the free surface is analyzed for the parabolic crack front that fits the actual geometry observed on the postmortem photograph. In Figure 10(a), stress intensity factors are normalized by the value for the mid-plane and the straight front. It is observed that the crack front geometry has a strong influence not only on the mean value of $K_{I}$ but also on its dependence with the domain size.

Further, opposite tendencies are obtained for the three different cases, namely, for the analysis on the mid-plane of a crack with a straight front, $K_{I}$ is almost constant and is chosen as a reference value $K_{I o}$, on the free surface the value of $K_{I}$ increases from 0.95 to 0.97 the reference value $K_{I o}$ whereas for the curved front $K_{I}$ decreases from 1.11 to 1.09. The same trend is obtained for the shift and the process zone width. A 0 shift is obtained for the straight front for the examined section but a shift of about $-20 \mu \mathrm{m}$ 
is obtained for the parabolic crack front (Figure 10(c)), meaning that the depth of influence of the front geometry is about $350 \mu \mathrm{m}$ (or 165 pixels), which is about 0.12 times the specimen thickness, and 0.07 times its width for an apparent crack length of 0.5 times its width.

Even though the previous analysis may only be qualitative, it is proven that $3 \mathrm{D}$ effects may exhibit supersingular contributions. Compared with the results obtained in the previous paragraph for the experimental specimen, the same trends are obtained, namely, the crack tip position is estimated in front of its emergence on the free surface due to curvature effects and a process zone size of about 40 to $50 \mu \mathrm{m}$ is estimated.

A mask is used to select the domain over which displacements are used in the analysis. The outer boundary of the mask is now chosen to be the largest disk included inside the region of interest. In addition, the immediate vicinity of the crack faces is excluded from the analysis as well as a small disk of radius $r_{0}$ centered on a rough prior determination of the crack tip. Figure 11 shows the influence of this inner radius $r_{0}$. It is observed that this excluded crack tip region has a significant influence as could have been anticipated by the singular nature of the fields at the crack tip. However, remarkably, for $r_{0}$ less than about $175 \mu \mathrm{m}$, no systematic trend is observed (although fluctuations are present). 


\subsubsection{SIF extraction}

In this last paragraph, the gain brought by an estimation not only of the displacement field but also of its discontinuity is evaluated. The analysis is carried out using the crack tip position determined in the previous paragraph. First, orthogonality conditions with K \& M "harmonics" in the interval $[-3 ; 5]$ are prescribed. The mode $I$ stress intensity factors measured using least squares technique and the proposed optimal extraction are shown in Figure 12. $K_{I}$ is plotted as a function of the diameter of the circular integration domain around the crack tip. A value of $3.1 \mathrm{MPa} \sqrt{\mathrm{m}}$ is obtained. The RMS error for an image noise of 2 gray levels is displayed with error bars in Figure 12. For the largest integration domain, the uncertainty reduces to $0.02 \mathrm{MPa} \sqrt{\mathrm{m}}$ for the optimal extraction whereas it is equal to $0.05 \mathrm{MPa} \sqrt{\mathrm{m}}$ when the least squares technique is applied.

These results are compared to those obtained in Ref. [24] when using a standard or optimized interaction integral. Figure 13(a) shows the change of $K_{I}$ for four extraction techniques, namely, least squares and optimal techniques, interaction integral with a conical virtual crack extension field (VCE) and interaction integral with an optimal VCE. Although the interaction integral is theoretically domain independent [17], larger variations with the domain size are observed due to a higher sensitivity to noise. Yet, the values obtained with the four techniques are consistent for large integration domains, namely, $2.9 \pm 0.09 \mathrm{MPa} \sqrt{\mathrm{m}}$ and $3.0 \pm 0.06 \mathrm{MPa} \sqrt{\mathrm{m}}$ for the interaction integral with conical and optimal VCEs. 
Figure 13(b) shows the standard uncertainty for $K_{I}$ with respect to the domain size. Power law decreases are obtained for the four techniques. The least squares and the optimal extractions vary as $D^{-2}(D$ being the diameter of the integration domain) but they differ from the optimal interaction integral $D^{-1.5}$ and the standard interaction integral $D^{-1}$. In Ref. [24], the $D^{-1.5}$ decrease is shown to be the optimal rate for the interaction integral. Let us note that the formalism proposed herein allows for higher reduction rates of noise sensitivity (i.e. $D^{-2}$ ). This is due to the fact that differential operators are involved in the interaction integral and reduce the noise reduction rate of any derived extractor. The difference between least squares and optimal extraction appears to be a multiplicative constant only.

Figures 14 and 15 show the extracting fields of mode $I$ stress intensity factors for the four above mentioned techniques. The least squares extracting field (Figure 14(b)) gives a considerable weight to the displacement values very close to the crack tip. The optimal version spreads the weight over a larger neighborhood, and accounts also for the non-homogeneous noise sensitivity of the displacement field measurement (Figure 14(a)). This effect allows for a lower noise sensitivity of the extracted stress intensity factor $K_{I}$. By looking at the extracting fields related to the interaction integrals, a considerable weight is carried by the displacement values not only close to the crack tip but also along the crack faces (Figure 15(b)). Further, a flower shape around the crack tip is observed. Because of the presence of differential operators in the integration integral, the shape of the extracting field is more 
tortuous and concentrated around the crack tip. Again, the optimal version spreads the weight over a larger neighborhood (Figure 15(a)).

Due to the very brittle nature of the ceramic, one may think of relaxing the orthogonality conditions with respect to supersingular modes. As a consequence, the optimal field becomes more uniform allowing all displacement degrees of freedom to contribute to the determination of the displacement field. Figure 16(a) shows the corresponding extracting field. Concerning the least squares extracting field, Figure 16(b) also shows less concentrated weighting but its noise sensitivity increases significantly (Figure 17(b)). Consequently, $K_{I}$ values are not consistent with previous determinations (Figure 17(a)), whereas the optimal extraction with reduced orthogonality constrains gives consistent $K_{I}$ values of $3.1 \mathrm{MPa} \sqrt{\mathrm{m}}$ with a very low uncertainty level, namely $0.01 \mathrm{MPa} \sqrt{\mathrm{m}}$. This is presumably another illustration of the above discussed $3 \mathrm{D}$ effects.

\section{Conclusions}

A method devoted to the extraction of Fracture Mechanics parameters from a displacement field is derived in a general formalism. It is shown that the proposed framework encompasses least squares techniques as well as domain (interaction) integrals for stress intensity factor extraction. Applied to experimental (i.e. noisy) displacement data, the present formalism includes noise sensitivity reduction and extraction conditions. The resolution is achieved by minimizing the effect of noise under orthogonality constrains 
to a family of crack tip fields, including supersingular fields. Despite their energy diverging behavior, the latter ones are useful in an experimental context. They are used to obtain the crack tip position.

Two experimental applications have been proposed. In the first one, the small thickness of the specimen legitimates the use of a two-dimensional description, and the results are shown to improve the robustness and accuracy of the measurements of fracture properties. In the second example, although the large scale results seem consistent, a more pronounced domain dependence still remains, which is attributed to three dimensional effects. In fact, this difficulty is a major one, as far as the measurement itself relies on a description that is not exact. The $2 \mathrm{D}$ analysis is however used here in order to conform with the standard practice, yet a better suited field (as well as an amplitude equivalent to a SIF that can be quantitatively used) should be more thoroughly investigated.

The performances of the proposed optimal extraction are illustrated and compared to existing techniques through two examples. A significant improvement of the noise sensitivity is obtained by reaching a global minimizer of the effect of noise instead of a local one (for least squares or interaction integral techniques). Domain independence is obtained for stress intensity factors in spite of the mechanically non-admissible nature of measured displacement fields i.e. they do not satisfy the balance of momentum equation. Domain independence is also obtained for supersingular components but over a reduced domain size range. 
Examining domain integral approaches for stress intensity factor extraction within the framework proposed herein, unexpected results are obtained. Re-read as an extracting field, the interaction integral extracts stress intensity factors predominantly using displacement data at the nodes located close to the crack faces. In the present examples, it is emphasized by the use of a conical virtual crack extension fields. More regular virtual crack extension

fields could be used [19] to enlarge the useful data area around the crack tip, yet concentrated weights still remain along the crack faces.

Last, it is to be emphasized that the general procedure presented herein to compute optimal extractors is also suited to dealing not only with uncertain data, but also with kinematic fields being defined on arbitrary shape and topology domains, a property that is not found for, say, interaction integrals.

\section{Acknowledgments}

This work was funded by a CETIM Foundation grant entitled PROPAVANFIS: Advanced methods for the experimental and numerical analyses of crack propagations under complex loadings, and by Agence Nationale de la Recherche through grant RUPXCUBE (ANR-09-BLAN-0009-01). 


\section{References}

[1] M. Attigui and C. Petit, Mixed-mode separation in dynamic fracture mechanics: New path independent integrals, Int. J. Fract. 84 [1] (1997) 19-36.

[2] S. Avril, M. Grédiac and F. Pierron, Sensitivity of the virtual fields method to noisy data, Comput. Mech. 34 [6] (2004) 439-452.

[3] G. Besnard, F. Hild and S. Roux, "Finite-element" displacement fields analysis from digital images: Application to Portevin-Le Châtelier bands, Exp. Mech. 46 (2006) 789-803.

[4] H. D. Bui, Associated path independent J -integral for separating mixed modes, J. Mech. Phys. Solids 31 (1983) 439-448.

[5] H. K. Chen and R. T. Shield, Conservation Laws in Elasticity of the JIntegral Type, Journal of Applied Mathematics and Physics 28 (1977) $1-22$.

[6] P. Forquin, L. Rota, Y. Charles and F. Hild, A Method to Determine the Toughness Scatter of Brittle Materials, Int. J. Fract. 125 [1] (2004) 171-187.

[7] M. Goz, J. Dolbow and B. Moran, Domain integral formulation for stress intensity factor computation along curved three-dimensional interface cracks, Int. J. Solids Struct. 35 [15)] (1998) 1763-1783. 
[8] A. A. Griffith, The Phenomenon of Rupture and Flow in Solids, Phil. Trans. Roy. Soc. London A221 (1920) 163-198.

[9] R. Hamam, F. Hild and S. Roux, Stress intensity factor gauging by digital image correlation: Application in cyclic fatigue, Strain 43 (2007) 181-192.

[10] C. Henninger, S. Roux and F. Hild, Enriched kinematic fields of cracked structures, Int. J. Solids Struct. 47 (2010) 3305-3316.

[11] F. Hild and S. Roux, Digital image correlation: From measurement to identification of elastic properties - A review, Strain 42 (2006) 69-80.

[12] J. M. Huntley and J. E. Field, Measurement of crack tip displacement field using laser speckle photography, Eng. Fract. Mech. 30 [6] (1989) 779-790.

[13] G. R. Irwin, Analysis of the Stresses and Strains near the End of a Crack Traversing a Plate, ASME J. Appl. Mech. 24 (1957) 361-364.

[14] S. R. McNeill, W. H. Peters and M. A. Sutton, Estimation of stress intensity factor by digital image correlation, Eng. Fract. Mech. 28 [1] (1987) 101-112.

[15] N. Moës, J. Dolbow and T. Belytschko, A finite element method for crack growth without remeshing, Int. J. Num. Meth. Eng. 46 [1] (1999) 133-150. 
[16] N. Moës, A. Gravouil and T. Belytschko, Non-planar 3D crack growth by the extended finite element and level sets. Part I: Mechanical model, Int. J. Num. Meth. Eng. 53 [11] (2002) 2549-2568.

[17] D. M. Parks, A stiffness derivative finite element technique for determination of crack tip stress intensity factors, Int. J. Fract. 10 (1974) 487-502.

[18] D. M. Parks, The virtual crack extension method for nonlinear material behavior, Comp. Meth. Appl. Mech. Eng. 12 (1977) 353-364.

[19] H. Rajaram, S. Socrate and D. M. Parks, Application of domain integral methods using tetrahedral elements to the determination of stress intensity factors, Eng. Fract. Mech. 66 [5] (2000) 455-482.

[20] J. Réthoré, G. Besnard, G. Vivier, F. Hild and S. Roux, Experimental investigation of localized phenomena using Digital Image Correlation, Phil. Mag. 88 [28-29] (2008) 3339-3355.

[21] J. Réthoré, A. Gravouil, F. Morestin and A. Combescure, Estimation of mixed-mode stress intensity factors using digital image correlation and an interaction integral, Int. J. Fract. 132 (2005) 65-79.

[22] J. Réthoré, F. Hild and S. Roux, Shear-band capturing using a multiscale extended digital image correlation technique, Comp. Meth. Appl. Mech. Eng. 196 [49-52] (2007) 5016-5030. See also, J. Réthoré, F. Hild and S. 
Roux, Extended digital image correlation with crack shape optimization, Int. J. Num. Meth. Eng. 73 [2] (2008) 248-272.

[23] J. Réthoré, S. Roux and F. Hild, From pictures to extended finite elements: Extended digital image correlation (X-DIC), C. R. Mécanique 335 (2007) 131-137.

[24] J. Réthoré, S. Roux and F. Hild, Noise-robust Stress Intensity Factor Determination from Kinematic Field Measurements, Eng. Fract. Mech. 75 [13] (2008) 3763-3781.

[25] J. Réthoré, S. Roux and F. Hild, An extended and integrated digital image correlation technique applied to the analysis fractured samples, Eur. J. Comput. Mech. 18 (2009) 285-306.

[26] J. R. Rice, Mechanics of crack tip deformation and extension by fatigue, Proceedings Fatigue crack propagation, STP 415, (ASTM, Philadelphia (USA), 1967), 247-309.

[27] J. R. Rice, A Path Independent Integral and Approximate Analysis of Strain Concentrations by Notches and Cracks, ASME J. Appl. Mech. 35 (1968) 379-386.

[28] S. Roux and F. Hild, Stress intensity factor measurements from digital image correlation: Post-processing and integrated approaches, Int. J. Fract. 140 [1-4] (2006) 141-157. 
[29] H. W. Schreier, J. R. Braasch and M. A. Sutton, Systematic errors in digital image correlation caused by intensity interpolation, Opt. Eng. 39 [11] (2000) 2915-2921.

[30] M. A. Sutton, S. R. McNeill, J. Jang and M. Babai, Effects of subpixel image restoration on digital correlation error estimates, Opt. Eng. 27 [10] (1988) 870-877.

[31] M. L. Williams, On the stress distribution at the base of a stationary crack, ASME J. Appl. Mech. 24 (1957) 109-114.

[32] S. Yoneyama, T. Ogawa and Y. Kobayashi, Evaluating mixed-mode stress intensity factors from full-field displacement fields obtained by optical methods, Eng. Fract. Mech. 74 (2007) 1399-1412.

[33] O. C. Zienkievicz and R. L. Taylor, The Finite Element Method, (McGraw-Hill, London (UK), 4th edition, 1989). 


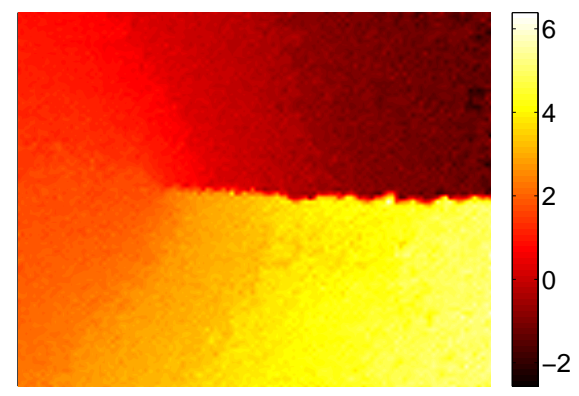

(a)

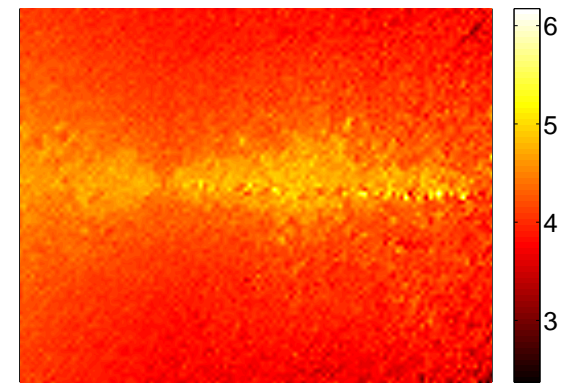

(b)

Figure 1: Vertical (a) and horizontal (b) components of measured displacement field by Q4-DIC for the CCT specimen, expressed in pixels (1 pixel $\leftrightarrow 2.08 \mu \mathrm{m}$ ). 


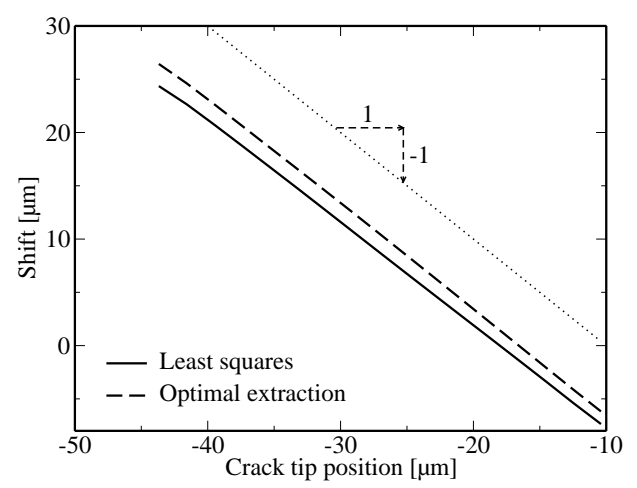

(a)

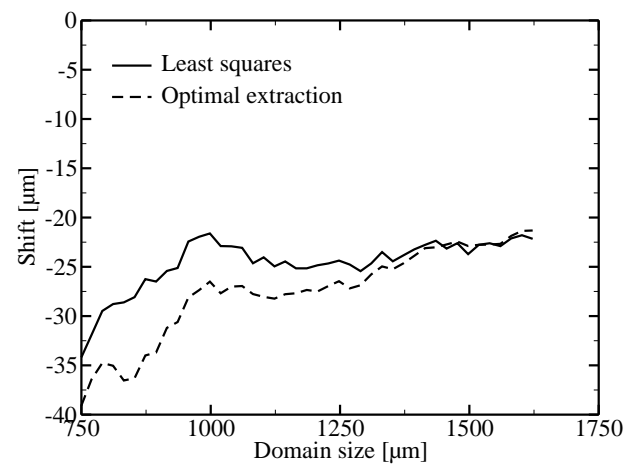

(b)

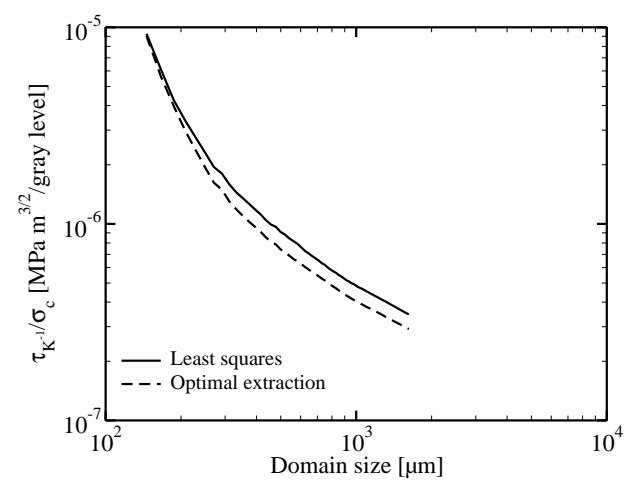

(c)

Figure 2: (a): Crack tip shift as a function of the crack tip position for the CCT specimen obtained with a least squares and optimal extraction performed with an $L_{2}$ projection and orthogonality conditions with $\mathrm{K} \& \mathrm{M}$ harmonics in $[-3 ; 5]$ (a dotted line of slope -1 is shown as a guide to the eye). (b): Crack tip shift as a function of the domain size. (c): noise sensitivity of the first supersingular coefficient. 


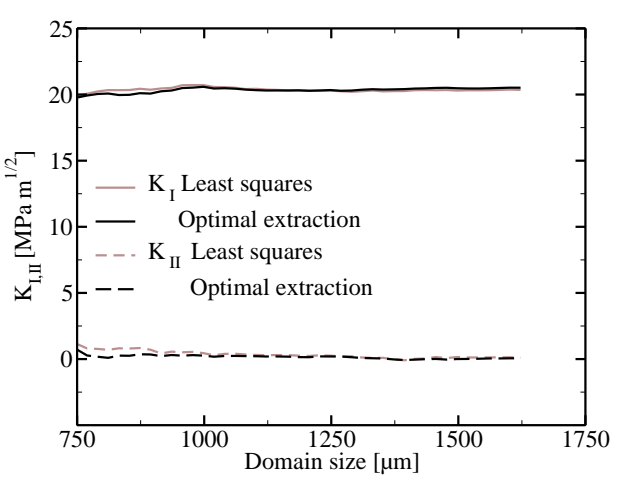

(a)

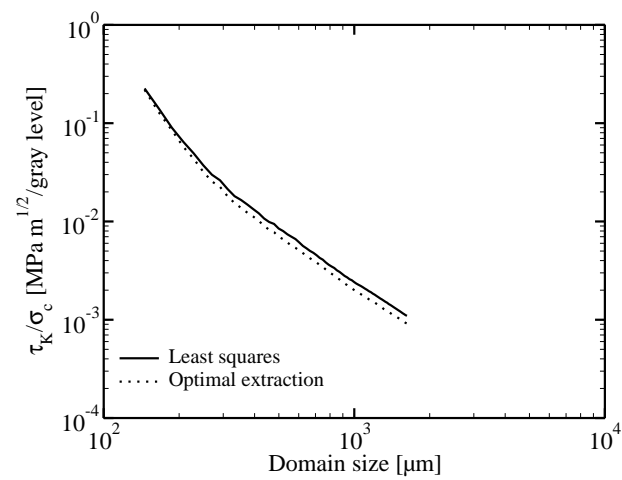

(b)

Figure 3: Stress intensity factors (a) and their noise sensitivity (b) for the CCT specimen obtained with a least squares and optimal extraction performed with an $L_{2}$ projection and orthogonality conditions with $\mathrm{K} \& \mathrm{M}$ harmonics in $[-3 ; 5]$. 

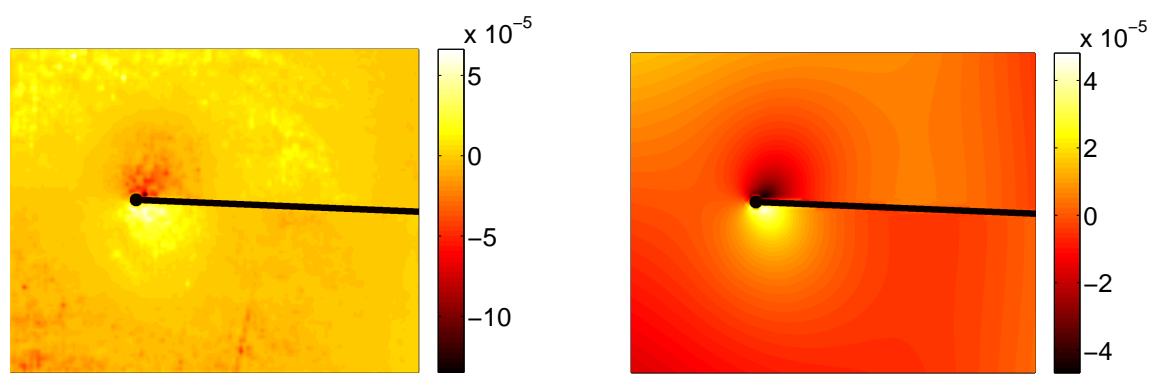

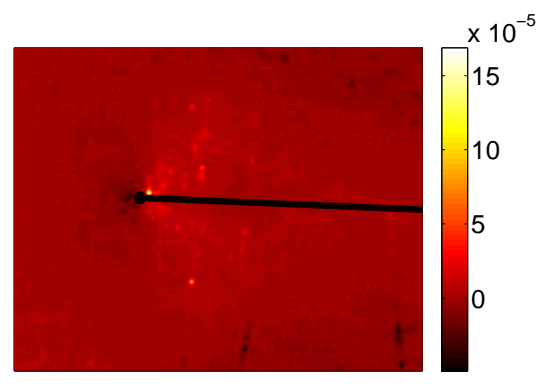

(a)

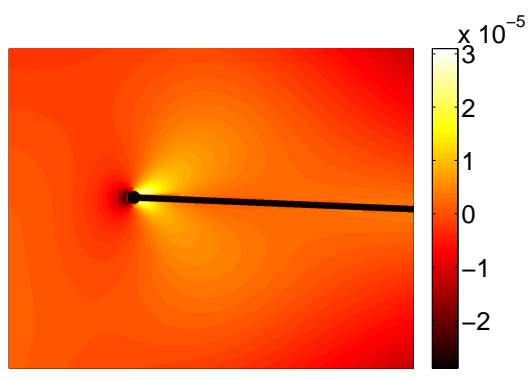

(b)

Figure 4: Vertical (top) and horizontal (bottom) components of the extracting field normalized by $2 \mu \sqrt{p \pi}$ obtained for an optimal extraction (left) a least squares extraction (right) of $K_{I}$ performed with an $L_{2}$ projection and orthogonality conditions with $\mathrm{K}$ \& $\mathrm{M}$ harmonics in $[-3 ; 5]$. 

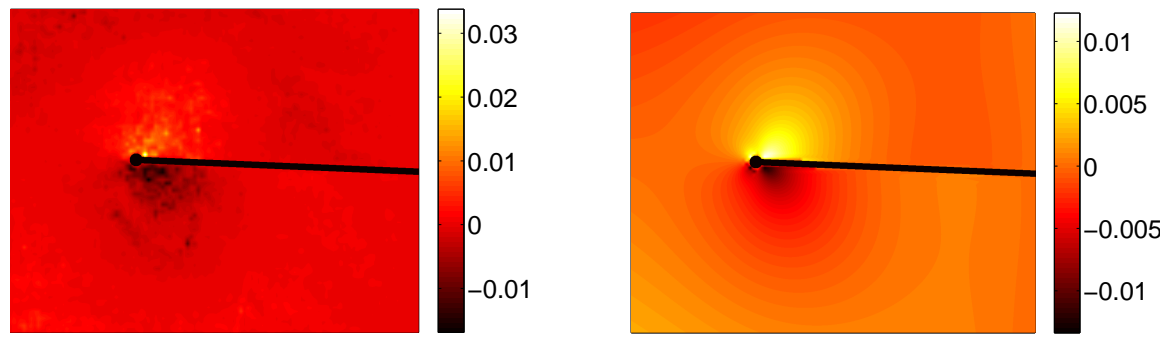

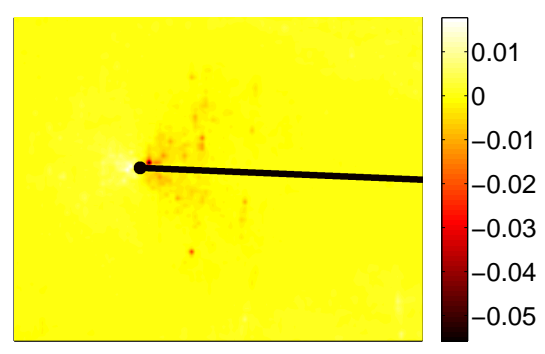

(a)

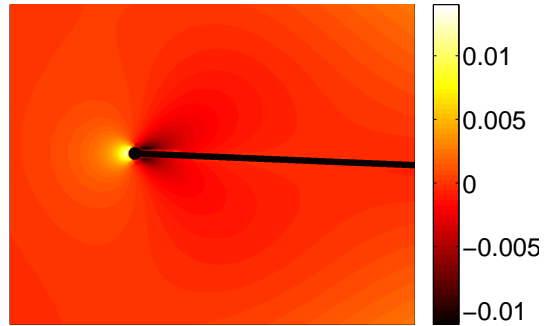

(b)

Figure 5: Vertical (top) and horizontal (bottom) components of the extracting field normalized by $2 \mu \sqrt{p^{3} \pi}$ obtained for an optimal extraction (left) a least squares extraction (right) of $K_{I}^{-1}$ performed with an $L_{2}$ projection and orthogonality conditions with $\mathrm{K} \&$ $\mathrm{M}$ harmonics in $[-3 ; 5]$. 


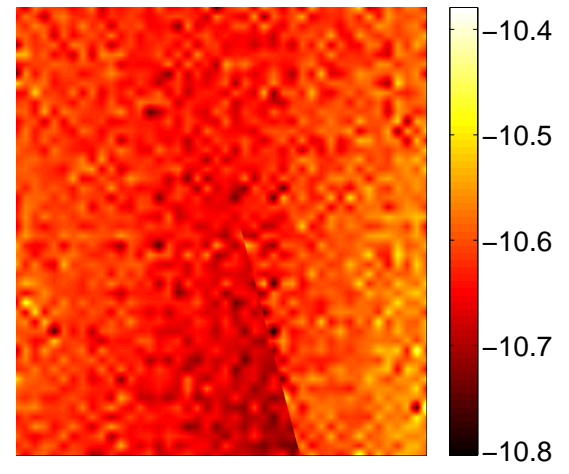

(a)

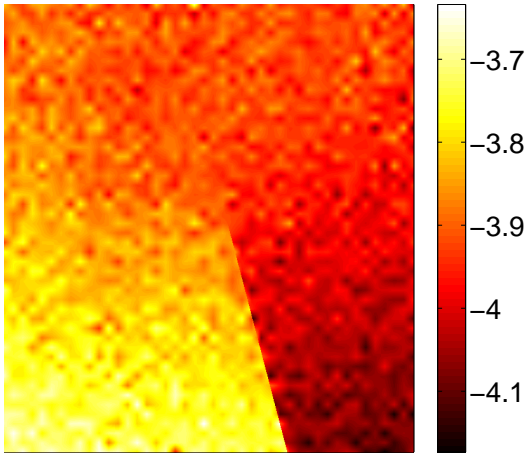

(b)

Figure 6: Vertical (a) and horizontal (b) components of the displacement field expressed in pixels measured by XQ4-DIC (1 pixel $\leftrightarrow 1.85 \mu \mathrm{m})$. 


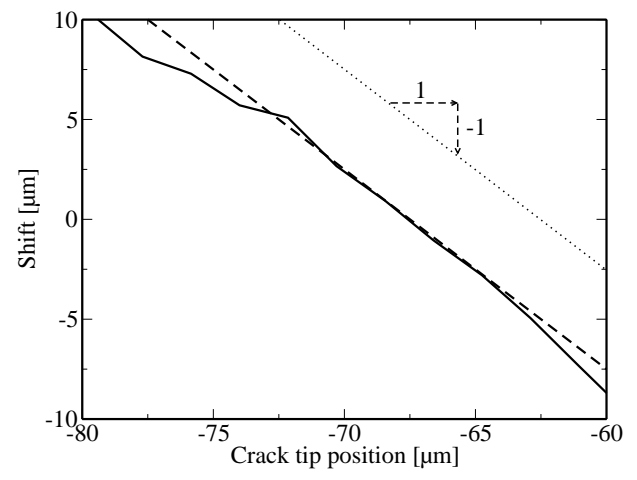

(a)

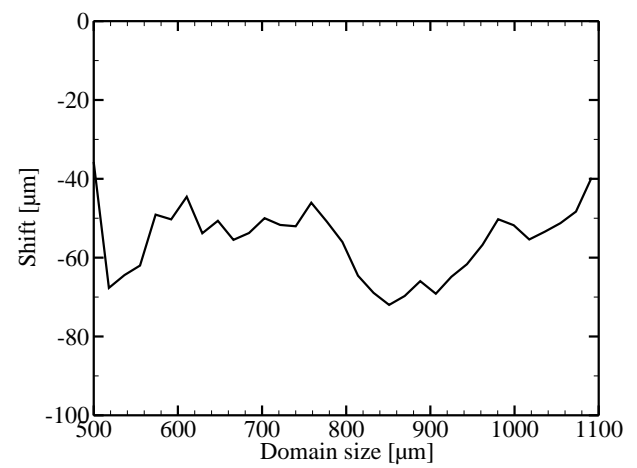

(b)

Figure 7: Crack tip shift as a fonction of the crack tip position (a) (a dotted line of slope -1 is shown as a guide to the eye) and as a function of the domain size (b) for the $\mathrm{SiC}$ specimen using the optimal extraction performed with an $L_{2}$ projection and orthogonality conditions with $\mathrm{K} \& \mathrm{M}$ harmonics in $[-3 ; 5](1$ pixel $\leftrightarrow 1.85 \mu \mathrm{m})$. 


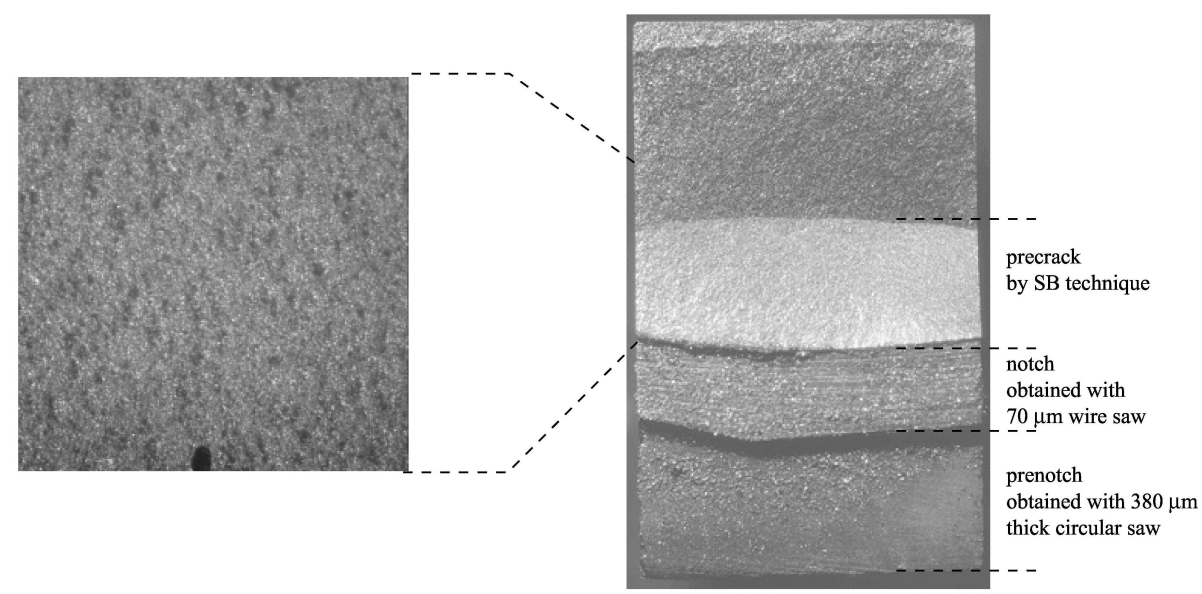

Figure 8: (left) Reference picture of the analyzed region of interest in the $\mathrm{SiC}$ sample (the fine notch can be seen on the bottom side). (right) post-mortem view of the crack surface. The pre-notch, the fine notch, and the precrack surfaces are clearly observable. Note the curvature of the precrack front. 

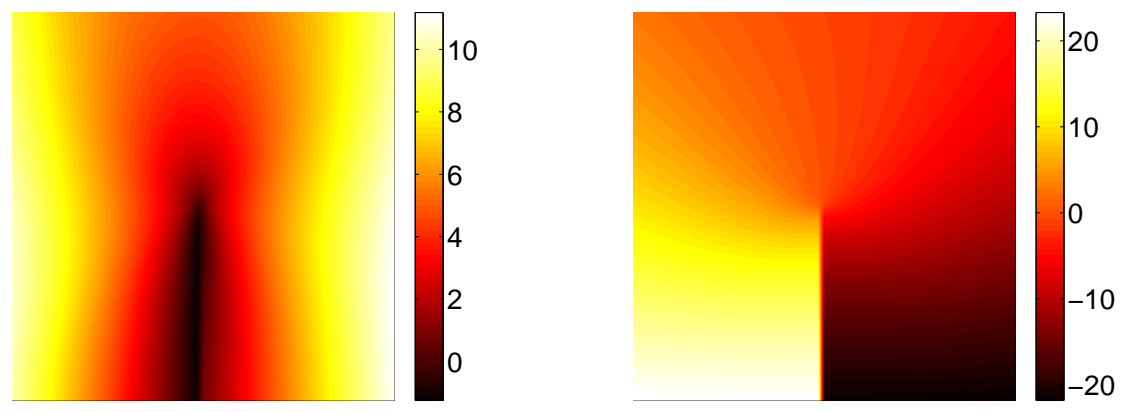

Figure 9: Vertical (a) and horizontal (b) components of the displacement field on the free surface of a three-dimensional elastic solid expressed in pixels obtained with a straight crack $(1$ pixel $\leftrightarrow 2.11 \mu \mathrm{m})$. 


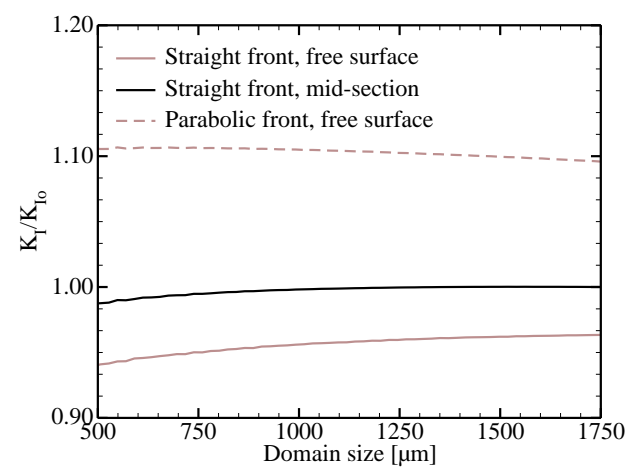

(a)

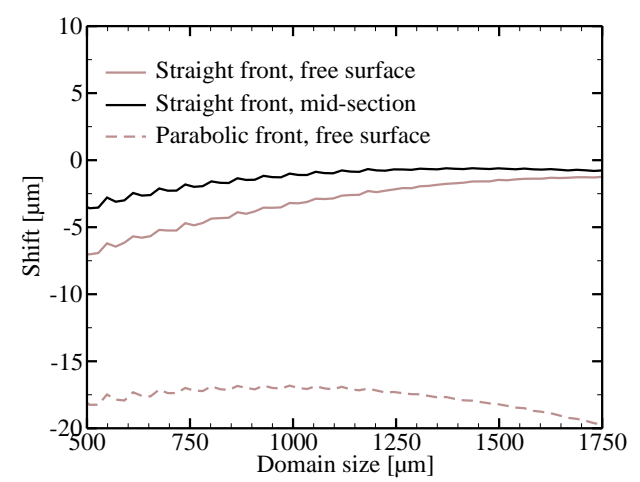

(b)

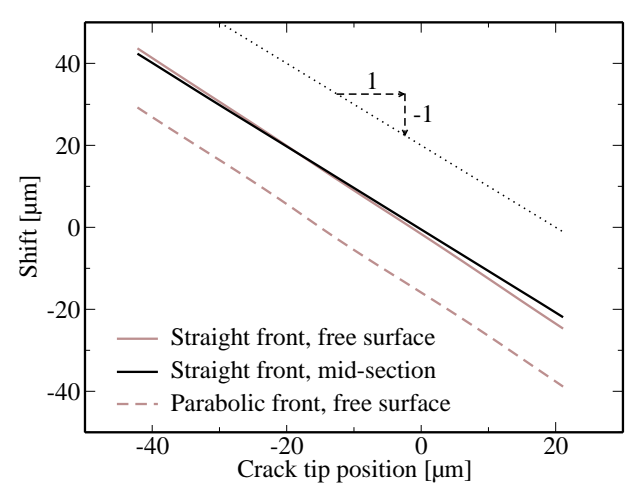

(c)

Figure 10: Normalized stress intensity factors (a) and shift (b) of the 3D elastic solid for a parabolic crack front, a straight front on the free surface and in the center section of the specimen $(1$ pixel $\leftrightarrow 2.11 \mu \mathrm{m})$. The crat 1 tip shift is also plotted as a fonction of the crack tip position (c) (a dotted line of slope -1 is shown as a guide to the eye). 


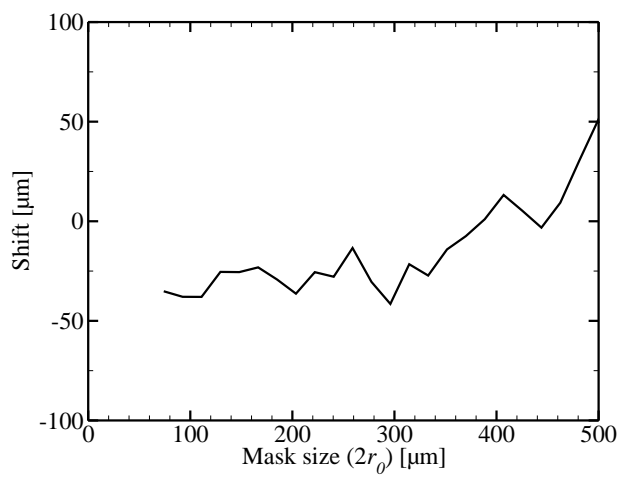

Figure 11: Influence of the mask inner diameter $2 r_{0}$ on the crack tip shift for the $\mathrm{SiC}$ specimen using the optimal extraction performed with an $L_{2}$ projection and orthogonality conditions with $\mathrm{K} \& \mathrm{M}$ harmonics in $[-3 ; 5]$ (1 pixel $\leftrightarrow 1.85 \mu \mathrm{m})$. 


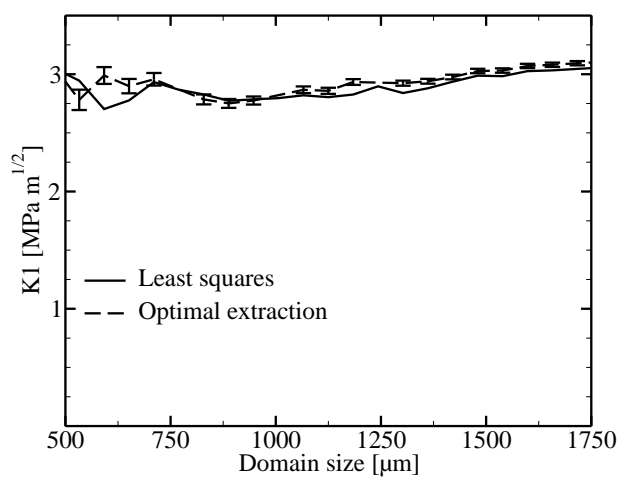

Figure 12: Comparison of mode $I$ stress intensity factors for the $\mathrm{SiC}$ specimen obtained with different extraction techniques. 


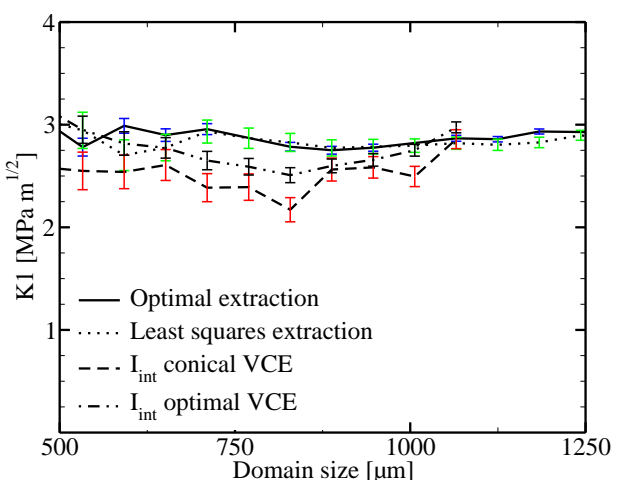

(a)

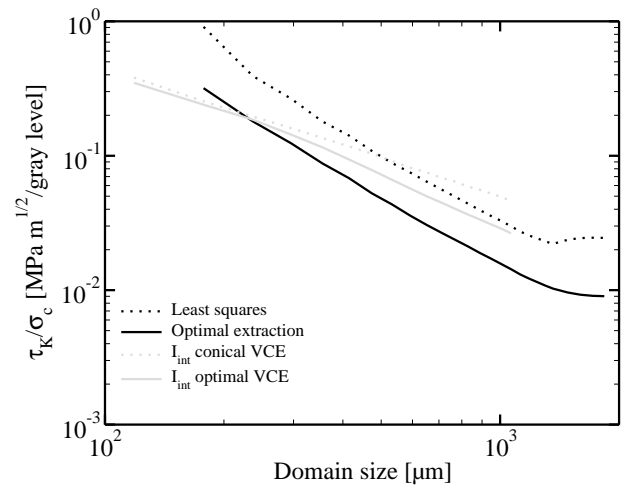

(b)

Figure 13: Comparison of mode $I$ stress intensity factors and noise sensitivities for the $\mathrm{SiC}$ specimen obtained with different extraction techniques including previous results with the interaction integral [24]. 

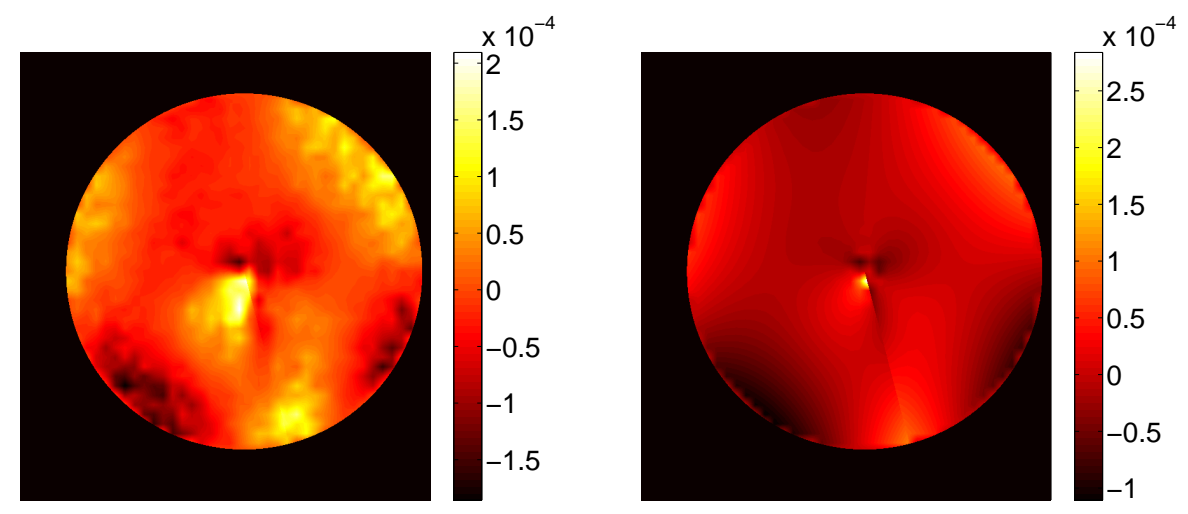

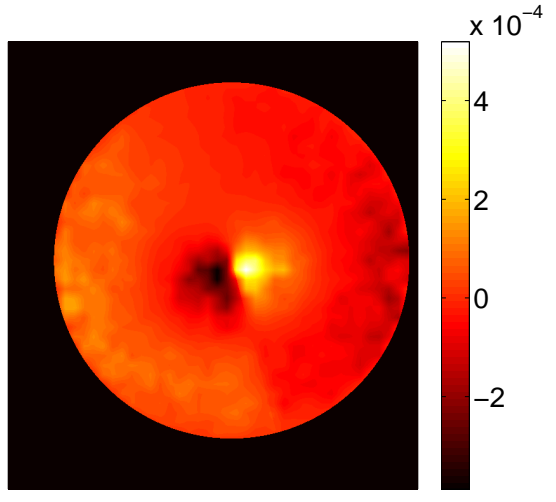

(a)

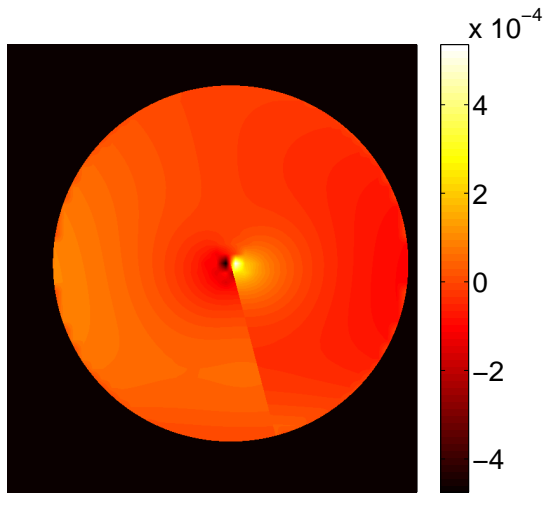

(b)

Figure 14: Vertical (top) and horizontal (bottom) components of the extracting field normalized by $2 \mu \sqrt{p \pi}$ obtained for an optimal (actual noise) (left) and a least squares (right) extraction of $K_{I}$ performed with an $L_{2}$ projection and orthogonality conditions with $\mathrm{K} \& \mathrm{M}$ harmonics in $[-3 ; 5]$. 

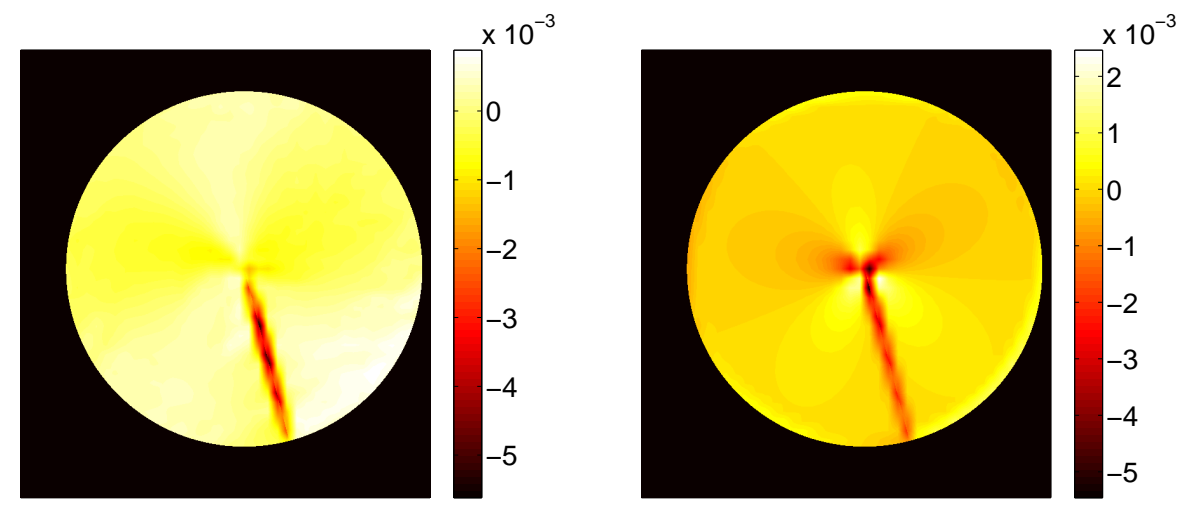

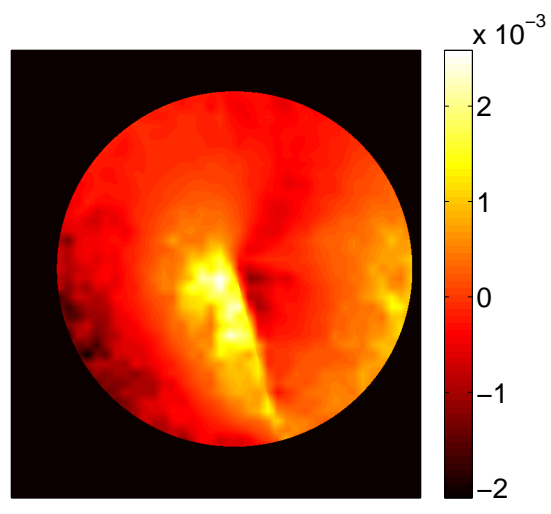

(a)

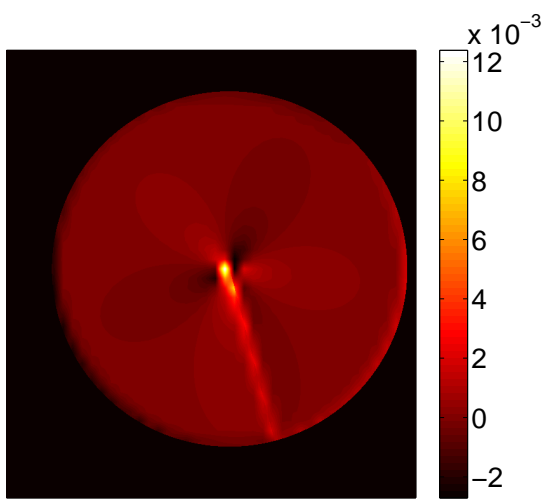

(b)

Figure 15: Vertical (top) and horizontal (bottom) components of the extracting field of $K_{I}$ normalized by $2 \mu \sqrt{p \pi}$ obtained for the interaction integral with an optimal VCE (left) and a conical VCE (right). 

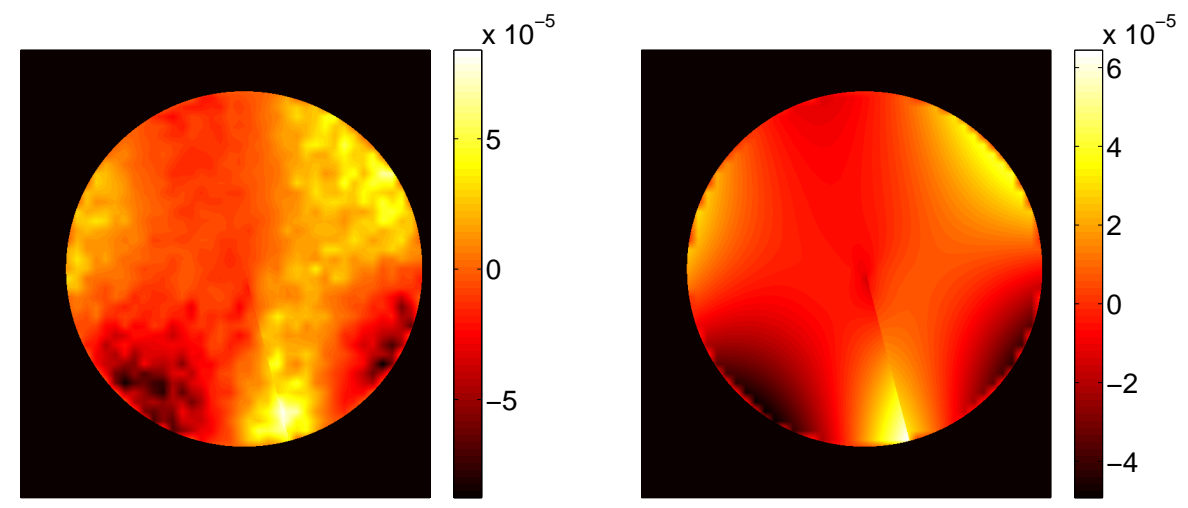

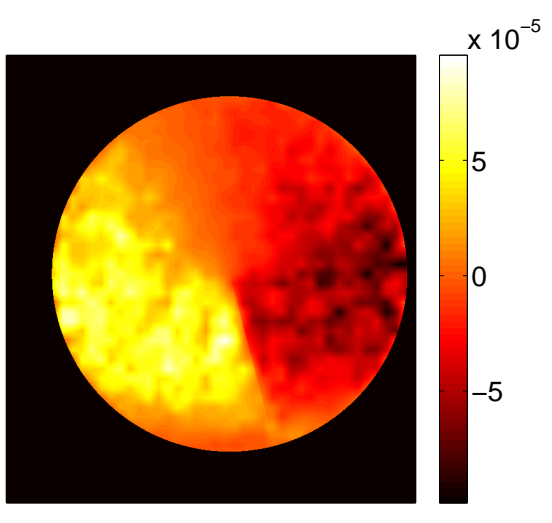

(a)

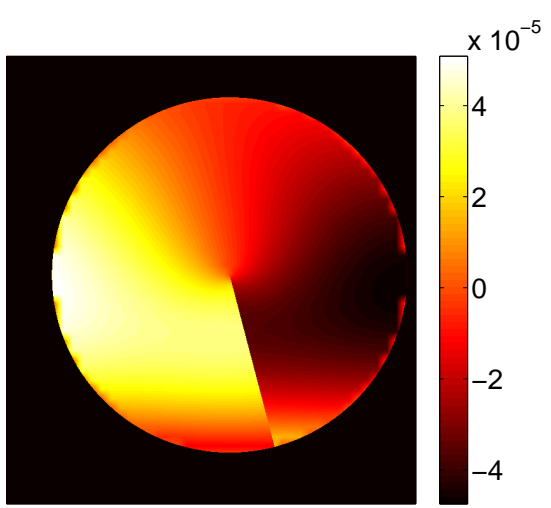

(b)

Figure 16: Vertical (top) and horizontal (bottom) components of the extracting field of $K_{I}$ normalized by $2 \mu \sqrt{p \pi}$ obtained for an optimal (actual noise) extraction performed with an $L_{2}$ projection and orthogonality conditions with $\mathrm{K} \& \mathrm{M}$ harmonics in $[0 ; 5]$. 


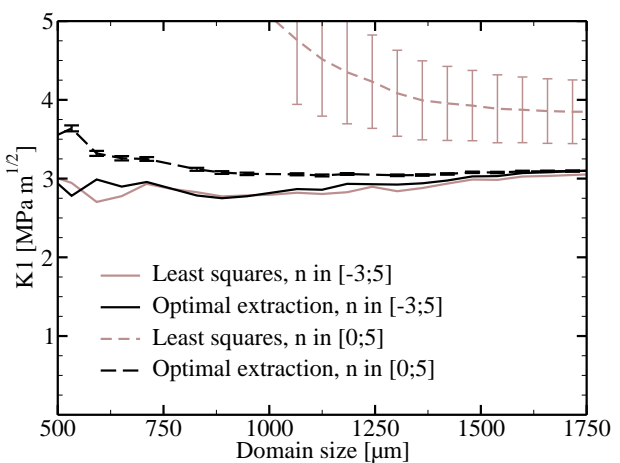

(a)

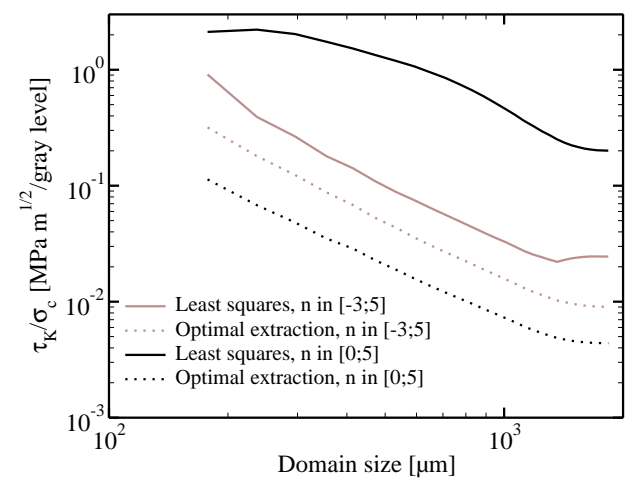

(b)

Figure 17: Comparison mode $I$ stress intensity factors (a) and noise sensitivities (b) for the $\mathrm{SiC}$ specimen obtained for orthogonality conditions prescribed for two different sets of K \& M harmonics. 